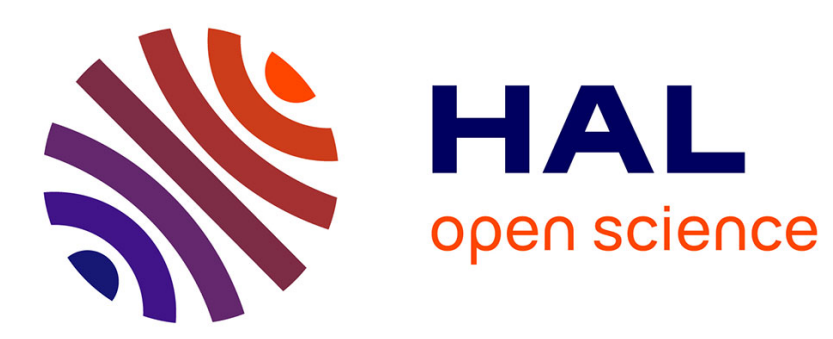

\title{
Heterodyne Holography: An Optimal and Versatile 2D Detection Scheme
}

\author{
Michel Gross
}

\section{To cite this version:}

Michel Gross. Heterodyne Holography: An Optimal and Versatile 2D Detection Scheme. Digital Holog- raphy \& 3-D Imaging, (Optical Society of America, May 2015, Shanghai, China. pp.DM2A.1., 10.1364/DH.2015.DM2A.1 . hal-01224128

\section{HAL Id: hal-01224128 \\ https://hal.science/hal-01224128}

Submitted on 4 Nov 2015

HAL is a multi-disciplinary open access archive for the deposit and dissemination of scientific research documents, whether they are published or not. The documents may come from teaching and research institutions in France or abroad, or from public or private research centers.
L'archive ouverte pluridisciplinaire HAL, est destinée au dépôt et à la diffusion de documents scientifiques de niveau recherche, publiés ou non, émanant des établissements d'enseignement et de recherche français ou étrangers, des laboratoires publics ou privés. 


\title{
Heterodyne Holography: An Optimal and Versatile 2D Detection Scheme
}

\author{
Michel Gross \\ Laboratoire Charles Coulomb (L2C), UMR 5221 CNRS-Université de Montpellier, Montpellier, F-France \\ michel.gross@univ-montp2.fr
}

\begin{abstract}
Heterodyne holography is a variant of phase shifting holography in which reference and signal arms are controlled by acousto optic modulators. We will briefly describe the method and illustrate its advantages on experimental examples.
\end{abstract}

OCIS codes: 090.1995: Holography: Digital holography, 040.2840: Detectors- Heterodyne

Citation M. Gross, "Heterodyne Holography: An Optimal and Versatile 2D Detection Scheme," in Digital Holography \& 3-D Imaging Meeting, OSA Technical Digest (Optical Society of America, 2015), paper DM2A.1.

\section{Introduction}

Heterodyne holography is a variant of phase shifting holography [1] introduced about 15 years ago [2] in which the frequency, phase and amplitude of both reference and signal beams are controlled by acousto optic modulators (AOM). By shifting the frequency $\omega_{L O}$ of the local oscillator beam with respect to the frequency $\omega_{I}$ of illumination, heterodyne holography is able to detect the light scattered by the object at a frequency $\omega$ close to $\omega_{L O}$, which can be different than the frequency $\omega_{I}$ of illumination of the object. To fully exploit this capability without being affected by a spurious detection at the alias frequency $\simeq 2 \omega_{I}-\omega_{L O}$ heterodyne holography must be combined with off-axis holography. This has been done in 2003 [3], and since.

\section{Setup and method}

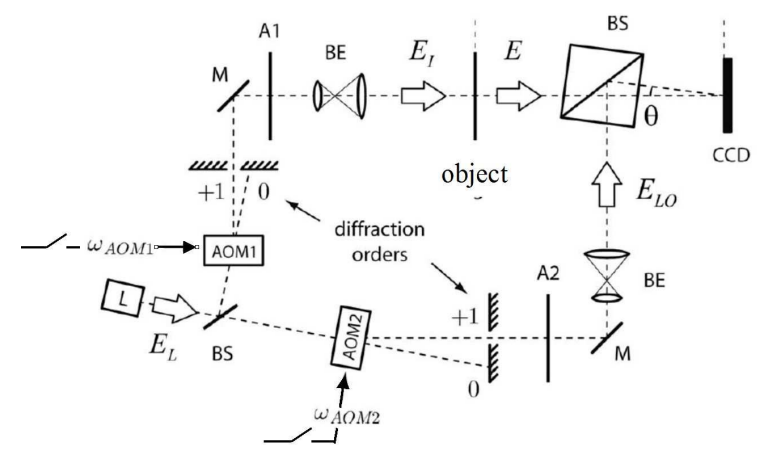

Fig. 1. Typical heterodyne holography setup in transmission geometry. L: main laser; BS: beam splitter; AOM1, AOM2: acousto-optic modulators; BE: beam expander; M, mirror; A1, A2: light attenuators; CCD: camera; $E_{L}, E_{I}, E_{L O}$, and $E$ : laser, illumination, reference (i.e. local oscillator LO) and object fields; $\omega_{A O M 1 / 2}$ : driving frequencies $(\simeq 80 \mathrm{MHz})$ of the acousto optics modulators AOM1 and AOM2; $\theta$ : off-axis angular tilt. where

Figure 1 shows a typical heterodyne holography setup. The frequency and the amplitude of both the illumination and references beams are controlled by the Radio Frequency RF signals at $\omega_{A O M 1}$ and $\omega_{A O M 2}$ with $\omega_{I}=\omega_{L}+\omega_{A O M 1}$ (illumination) and $\omega_{L O}=\omega_{L}+\omega_{A O M 2}$ (reference). An angularly tilted $(\theta \neq 0)$ beam splitter BS mixes the signal $(E)$ and reference $\left(E_{L O}\right)$ fields, and the interference pattern $I=\left|E+E_{L O}\right|^{2}$ is recorded by the CCD camera. Sequence of frames $I_{n}$ are acquired at frame frequency $\omega_{C C D}$. By adjusting the frequencies $\omega_{A O M 1,2}$, and by selecting the linear 
combination of frames $I_{n}$ (i.e. the demodulation equation) that are used to calculate the hologram $H$, different types of holographic detection schemes can be implemented.

- To detect the signal at the illumination frequency $\omega_{I}$ with 4 phases and $n_{\max }=4$ frames, we must have: $H=$ $\left(I_{0}-I_{2}\right)+j\left(I_{1}-I_{3}\right)$ and $\omega_{L O}=\omega_{I}+\omega_{C C D} / 4$ (i.e. $\left.\omega_{A O M 2}-\omega_{A O M 1}=\omega_{C C D} / 4\right)$.

- To make detection at $\omega \neq \omega_{I}$ with 2 phases and $n_{\max }$ frames, we must have similarly: $H=\sum_{n=0}^{n_{\max }}(-1)^{n} I_{n}$ and $\omega_{L O}=\omega+\omega_{C C D} / 2$ (i.e. $\omega_{A O M 2}-\omega_{A O M 1}=\omega-\omega_{I}+\omega_{C C D} / 2$ ).

\section{Advantages and examples}
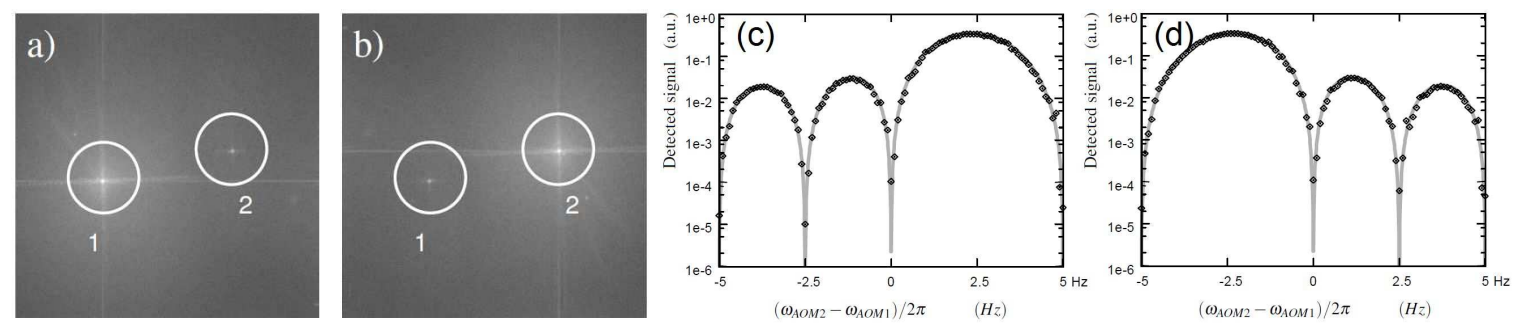

Fig. 2. (a,b) Fourier space holograms $|\tilde{H}|^{2}$ recorded with $\omega_{A O M 2}-\omega_{A O M 1}=+\omega_{C C D} / 4$ (a) and $-\omega_{C C D} / 4$ (b). (c,d) Sum of the detected signal $|\tilde{H}|^{2}$ in region 1 (c) and 2 (d) versus $\omega_{A O M 2}-\omega_{A O M 1}$ : measurement (points) and theory (solid grey line). See [4].

Heterodyne holography exhibit many advantages. First, phase shifting is made with a very accurate phase [4]. The hologram can thus be calculated without having to take account of the phase defects of the experimental setup. The frequency response of the holographic detection can thus be calculated and agrees with calculation. Figure 2 (a) and (b) shows the Fourier hologram $\tilde{H}=\operatorname{FFT}(H)$ of a USAF target obtained with $H=\left(I_{0}-I_{2}\right)+j\left(I_{1}-I_{3}\right)$ and $\omega_{A O M 2}-\omega_{A O M 1}=+\omega_{C C D} / 4$ (a) and $-\omega_{C C D} / 4$ (b). Peak 1 corresponds to the transmitted ballistic light detected by 4 phase detection at $\omega_{L O}-\omega_{C C D} / 4$, while peak 2 is the spurious alias at $\omega_{L O}+\omega_{C C D} / 4$. We have plotted on Fig. 2 (c) and (d) the frequency response of the holographic detection made on peak 1 (c), and peak 2 (d). Experiment (points) agrees with theory (solid grey line).

As it can be seen in Fig. 2, heterodyne holography performs the detection at the two sideband frequencies $\omega_{L O} \mp$ $\omega_{C C D} / 4$ that correspond to 4 phases demodulation. The off-axis configuration of Fig.1 allows to separate these 2 sidebands in the Fourier space (peak 1 and 2 are separated). It is then possible to select one of the two sideband, avoiding thus frequency alias.

The second advantage is selectivity and sensitivity. In Fig. 2 (a), the useful signal is peak 1. It is extracted in the recorded data $\left(I_{0} \ldots I_{3}\right)$ by a double filtering procedure. The hologram $H$ is calculated by $H=\left(I_{0}-I_{2}\right)+j\left(I_{1}-I_{3}\right)$. This means, that the time frequency component $\omega_{L O}-\omega_{C C D} / 4$ is selected in the data. Then one calculates $\tilde{H}=\mathrm{FFT}(H)$, and one select the spatial frequency components near peak 1 . This double filtering procedure is very efficient to filter off the zero and twin images grating order aliases. The technical noise thus cancel, and detection can easily reach shot noise sensitivity [5]. Lesaffre et al. [6] have shown that the total amount of noise (for all pixels of the reconstructed image) is $A$ photo electrons, where $A$ is the area in pixel Units of the Cuche et al. spatial filter [7]. The noise do not thus depends on the number $n_{\max }$ of frames $I_{n}$ used to calculate $H$, while the detected signal is proportional to $n_{\max }$. One must also mention that the noise floor related to shot noise can be used to calibrate the signal that is detected by holography $[8,9]$.

The third advantage is the ability of detecting a signal at a frequency different from that illumination. This point splits in two sub points. In some cases, the object moves and the scattered light exhibit a broads continuous frequency spectrum, which can be analyzed by sweeping $\omega_{A O M 1}$ or $\omega_{A O M 2}$ (in order to sweep the detection frequency $\omega$ ). The method is called Laser Doppler holography [10] and has been applied to image blood flow in mouse crania [11,12], rat eye [13] and fish embryo [14]. The method has been also used to analyse the broadening of the light that travel through a women breast in strong multiple scattering condition [8]. In that case, the signal is very week and corresponds to less than 0.1 photo electron per pixel at maximum. The object that is imaged to detect the signal is not the breast, but 
a rectangular slit (of $5 \mathrm{~mm}$ width) that is back illuminated by the light that travel through the breast. The measurement is made by comparing the signal detected in and outside the slot.

In some other cases, the objet vibrates at a fixed frequency $\omega_{A}$, and the scattered light exhibits frequency sidebands that can be detected selectively by heterodyne holography. The method is then called Sideband Digital holography [15]. It is more efficient than time averaged holography [16] that makes the holographic detection at the carrier frequency( $\omega=\omega_{I}$ ), especially for studying vibrations of large [17] and small amplitude [18, 19]. We must also mention that the RF signals that drive the AOMs can be used to switch on an off both illumination and detection in order to combine sideband holography with strobe illumination and detection. This stroboscopic sideband holography is sensitive to the phase of the vibration motion [20]. Vibration of extremely low amplitude can be also imaged in amplitude and phase by displaying the carrier versus first sideband signals correlation [21].

Acknowledgments. We acknowledge ANR Blanc Simi 10 ( ${ }^{\circ} 11$ BS10 015 02), ANR Blanc Simi 4 ( ${ }^{\circ} 11-B S 04$ 01704 ) and Labex Numev (convention ANR-10-LABX-20) grants for funding.

\section{References}

1. I. Yamaguchi and T. Zhang, "Phase-shifting digital holography,” Opt. Lett. 22, 1268-1270 (1997).

2. F. Le Clerc, L. Collot, and M. Gross, "Numerical heterodyne holography with two-dimensional photodetector arrays," Opt. Lett. 25, 716-718 (2000).

3. M. Gross, P. Goy, and M. Al-Koussa, "Shot-noise detection of ultrasound-tagged photons in ultrasoundmodulated optical imaging," Optics letters 28, 2482-2484 (2003).

4. M. Atlan, M. Gross, and E. Absil, "Accurate phase-shifting digital interferometry," Optics letters 32, 1456-1458 (2007).

5. M. Gross and M. Atlan, "Digital holography with ultimate sensitivity," Optics letters 32, 909-911 (2007).

6. M. Lesaffre, N. Verrier, and M. Gross, "Noise and signal scaling factors in digital holography in weak illumination: relationship with shot noise," Applied optics 52, A81-A91 (2013).

7. E. Cuche, P. Marquet, and C. Depeursinge, "Spatial filtering for zero-order and twin-image elimination in digital off-axis holography," Applied optics 39, 4070-4075 (2000).

8. M. Gross, P. Goy, B. Forget, M. Atlan, F. Ramaz, A. Boccara, and A. Dunn, "Heterodyne detection of multiply scattered monochromatic light with a multipixel detector," Optics letters 30, 1357-1359 (2005).

9. E. Absil, G. Tessier, M. Gross, M. Atlan, N. Warnasooriya, S. Suck, M. Coppey-Moisan, and D. Fournier, "Photothermal heterodyne holography of gold nanoparticles," Optics express 18, 780-786 (2010).

10. M. Atlan and M. Gross, "Laser doppler imaging, revisited," Review of Scientific Instruments 77, 116,103 (2006).

11. M. Atlan, M. Gross, B. C. Forget, T. Vitalis, A. Rancillac, and A. K. Dunn, "Frequency-domain wide-field laser doppler in vivo imaging," Optics letters 31, 2762-2764 (2006).

12. M. Atlan, B. C. Forget, A. C. Boccara, T. Vitalis, A. Rancillac, A. K. Dunn, and M. Gross, "Cortical blood flow assessment with frequency-domain laser doppler microscopy," Journal of biomedical optics 12, 024,019024,019 (2007).

13. M. Simonutti, M. Paques, J.-A. Sahel, M. Gross, B. Samson, C. Magnain, and M. Atlan, "Holographic laser doppler ophthalmoscopy," Optics letters 35, 1941-1943 (2010).

14. N. Verrier, D. Alexandre, and M. Gross, "Laser doppler holographic microscopy in transmission: application to fish embryo imaging," Optics express 22, 9368-9379 (2014).

15. F. Joud, F. LaloÚ, M. Atlan, J. Hare, and M. Gross, "Imaging a vibrating object by sideband digital holography," Optics express 17, 2774-2779 (2009).

16. P. Picart, J. Leval, D. Mounier, and S. Gougeon, "Time-averaged digital holography," Optics letters 28, 1900$1902(2003)$.

17. F. Joud, F. Verpillat, F. Laloë, M. Atlan, J. Hare, and M. Gross, "Fringe-free holographic measurements of large-amplitude vibrations," Optics letters 34, 3698-3700 (2009).

18. P. Psota, V. Ledl, R. Dolecek, J. Erhart, and V. Kopecky, "Measurement of piezoelectric transformer vibrations by digital holography," Ultrasonics, Ferroelectrics, and Frequency Control, IEEE Transactions on 59, 19621968 (2012).

19. N. Verrier and M. Atlan, "Absolute measurement of small-amplitude vibrations by time-averaged heterodyne holography with a dual local oscillator," Opt. Lett. 38, 739-741 (2013). 
20. F. Verpillat, F. Joud, M. Atlan, and M. Gross, "Imaging velocities of a vibrating object by stroboscopic sideband holography," Optics express 20, 22,860-22,871 (2012).

21. L. A. N. Verrier and M. Gross, "Vibration of low amplitude imaged in amplitude and phase by sideband versus carrier correlation digital holography," Opt. Lett. 40, 411-414 (2015). 